\title{
La escuela sociocultural de la paz pedagógica: Una apuesta hacia la construcción de paz sostenible
}

The socio-cultural school of educational peace: A commitment to building sustainable peace

A escola sociocultural da paz pedagógica: Uma aposta em direção a construção de paz sustentável

L'école socioculturelle de la paix pédagogique: Un pari vers la construction d'une paix durable 寓教于乐的社会文化学校:建设可持续的和平之间。

\section{María Inés Sánchez Cardona ${ }^{1}$ \\ Universidad Jaume I - España}

Revista Derechos en Acción ISSN 2525-1678/ e-ISSN 2525-1686

Año 5/Nº 14 VERANO 2019/20 (21 diciembre a 20 marzo), 197-223

DOI: https://doi.org/10.24215/25251678e357

ORCID: https://orcid.org/0000-0002-0718-6173

Recibido: 01/12/2019

Aprobado: 01/02/2020

Resumen: El constante desarrollo de nuevos estudios de paz ofrece siempre un marco o paradigma interpretativo de la realidad y específicamente de las expresiones individuales o sociales que pugnan en busca de ella. En esta perspectiva se sitúa la Escuela Sociocultural de la Paz Pedagógica, como una posibilidad teórica y práctica que intenta entender, transformar e interpretar nuevas realidades de la coexistencia

\footnotetext{
1 Mariela Sánchez Cardona, Doctora en Estudios de Paz Conflicto y Desarrollo de la Universidad Jaume I- España. Profesora investigadora a nivel de posgrados en diferentes universidades de Colombia, en temas de cultura y pedagogías de paz. Hace parte del grupo Constitucionalismo Comparado de la Universidad Nacional de Colombia donde lidera la línea de Constitucionalismo y Paz. Correo electrónico: marielainesanchez@hotmail.com
} 
en el mundo actual. Así pues, en el presente escrito se ponen en relación términos y acción como escuela, sociocultural y paz pedagógica como una propuesta hacía la cimentación de una paz sostenible.

Palabras clave: Escuela Sociocultural de la Paz Pedagógica, ética de la paz, cultura, sociedad.

Abstract: The constant development of new peace studies always offers a framework or interpretive paradigm of reality and specifically of the individual or social expressions that struggle in search of it. In this perspective the Sociocultural School of Pedagogical Peace is situated, as a theoretical and practical possibility offered to reality to understand, transform and interpret it. Thus, in this paper terms and action such as school, sociocultural and pedagogical peace are listed as a proposal towards the foundation of a sustainable peace.

Keywords: Escuela Sociocultural de la Paz Pedagógica, ethics of peace, culture, society.

Resumo: 0 constante desenvolvimento de novos estudos da paz oferece sempre uma quadro ou paradigma interpretativo da realidade e especificamente das expressões individuais ou sociais que lutam em busca dela. Nessa perspectiva está situada a Escola Sociocultural de Paz Pedagógica, como uma possibilidade teórica e prática que tenta entender, transformar e interpretar novas realidades de convivência no mundo atual. Assim, no presente artigo entram em relacionamento termos e ações como escola, sociocultural e paz pedagógica são como uma proposta para a fundação da paz sustentável.

Palavras-chave: Escola Sociocultural da Paz Pedagógica, ética da paz, cultura, sociedade.

Résumé: Le développement constant de nouvelles études sur la paix offre toujours un cadre ou un paradigme interprétatif de la réalité et tout particulièrement des expressions individuelles ou sociales qui luttent à sa recherche. L'École socioculturelle de la paix pédagogique se situe dans cette perspective, comme une possibilité théorique et pratique qui essaie de comprendre, transformer et interpréter les nouvelles réalités de la coexistence dans le monde d'aujourd'hui. Ainsi, dans le présent essai, des termes et action tels que école, socioculturelle et paix pédagogique sont mis en relation comme une proposition vers la fondation d'une paix durable. 
Mot-clés: Ecole Socioculturelle de la Paix Pédagogique, éthique de la paix, culture, société.

摘要: 新和平研究的不断发展总是为现实, 特别是为寻求现实而奋 斗的个人或社会表现形式提供解释框架或范例。教育和平社会文化 学派就以这种观点为出发点, 作为一种理论和实践可能性, 试图理 解, 转变和解释当今世界共存的新现实。因此, 在本写作中, 将诸如 学校，社会文化和教学和平之类的措辞和行动作为建立可持续和平 基础的建议。

关键词:教育和平社会文化学院, 和平伦理, 文化, 社会。

\section{Introducción}

Las acciones por generar escenarios de paz han sido una constante en el devenir del ser humano, su apuesta se ha caracterizado por abordar, desde lo individual, social, político, cultural y hasta económico, pensamientos que edifiquen una sociedad basada en principios que buscan empoderar la paz como un motor de la historia, hacer que aquella también forme parte de los manuales y libros de textos que muchas veces encarnan la historia oficial de las naciones (Jiménez Arenas \& Muñoz, 2012; Marquardt, 2017).

Así pues, la Escuela Sociocultural de la Paz Pedagógica estimula nuevos aprendizajes en torno al conocimiento e investigación de las paces como una apuesta hacia la construcción de una paz posible y sostenible en la humanidad. Al interior de ella se quiere resaltar tres conceptos que estarán siempre interactuando y retroalimentando los procesos: el concepto de escuela, de sociedad, donde está integrada la noción de cultura (sociocultural), y el concepto de paz pedagógica. En torno al primer concepto: la escuela, esta es concebida como un espacio humano donde se generan nuevos pensamientos $\mathrm{y}$ actuaciones frente a problemáticas sociales comunes para los actores (as) y participantes, los cuales pueden ser individuos o colectividades. A través de estos lineamientos se pretende 
en estos espacios tejer redes de conocimiento que acompañen y cuestionen constantemente la coherencia de paz en el pensamiento, sentimiento y actuación de la sociedad (Sánchez Cardona, 2016, 2019).

La segunda concepción -sociocultural-, tiene su relevancia en este saber ya que concebimos la violencia no como dimensión genética donde el ser humano y la sociedad no tiene opciones de vida, sino un aprendizaje sociocultural- tesis que es ampliamente argumentada por Adams en su texto Cultura de paz: una utopía posible (2014) -quien describe al ser humano bajo la posibilidad de imaginar nuevas formas de concebir y vivir la paz. En estos contextos socioculturales es donde se teje el lenguaje y tanto las cosmovisiones individuales como grupales de paz y/o violencia. Es al interior de estos escenarios, donde se desarrollan estrategias sostenibles de la paz basadas en las virtudes para la convivencia pacífica reconociendo y respetando la individualidad de cada cultura.

Con la tercera y última categoría, paz pedagógica, se quiere indicar que la paz es una construcción que se aprende (Ansembourg y Van Reybrouck: 2017), desaprende y se enseña todos los días, ella está presente en la interacción con nosotros mismos, el ambiente y la sociedad. Esta característica de ser pedagógica y transmisible permite el crecimiento y la investigación de nuevas formas de actuar en el mundo desde el saber y conocimiento de quien sufre y ha sido excluido socialmente. El aprendizaje con el otro(s) se concibe de forma horizontal, y se hace un giro del saber de la paz de centro a periferia. En este sentido, los académicos o los usualmente llamados expertos de paz y actores violentos no son el centro de estudio, sino los grupos y personas que viven o han vivido en diferentes condiciones la violencia (directa, estructural y/o cultural) (Jiménez, 2011; 2018) y desde allí han construido sus propias pedagogías para dignificar sus vidas en sus territorios o espacios socioculturales. De esta manera, la noción de paz pedagógica transversaliza el pensamiento de la Escuela Sociocultural y sirve de insumo constante para revitalizar y dar vida a las teorías de educación 
para la paz en diferentes contextos educativas. Al respecto, la autora ha planteado en otras investigaciones (2016, 2019), la importancia que las instituciones educativas de cada país desarrollen metodologías propias de paz para seguir reinventando y descubriendo otras formas de hacer las paces en una sociedad por ejemplo la colombiana, que ha sido atravesada por la violencia en diversos contextos sociopolíticos.

El presente artículo desarrollada la conceptualización epistémica y científica de la Escuela Sociocultural de la Paz Pedagógica en tres grandes apartados. El primero, expone algunas problemáticas que impiden la sostenibilidad de la paz en un mundo globalizado. El segundo apartado, desarrolla los principales horizontes y lineamientos estratégicos de la Escuela Sociocultural de la Paz Pedagógica. Por último, se presentan las conclusiones del estudio y las posibilidades de realización de los principios de la Escuela.

\section{Problemáticas que impiden la sostenibilidad de la paz en un mundo globalizado}

Las problemáticas socioculturales de las naciones entorno a la realización de una paz sostenible en el mundo, se ha convertido en un gran reto para los saberes académicos, epistemológicos, sociales, culturales y políticos de los Estados. Así pues, encontramos cifras alarmantes en el aumento en niveles de pobreza, desplazamiento forzado, recorte de presupuesto en programas sociales y educativos, altos niveles de corrupción, problemas de xenofobia, consumo desmedido, cambio climático, entre otros. Por ejemplo, en Honduras entre 2016 y 2019, según la Interpol, han desaparecido más de mil menores de edad y se calcula que el $70 \%$ de los niños y adolescente viven en condiciones de pobreza (Mesa, 2020). De igual forma, se observa como los niveles de violencia en Colombia han aumentado en los últimos años, según datos del Instituto de Medicina Legal en 2018 se registraron 12.130 homicidios, mientras que en 2019 se indican cerca de 11.800 (Forensis, 2019), así mismo 
sucede con el hurto, el desempleo, la escaza atención de salud y el elevado índice de suicidio.

En una entrevista realizada a la psiquiatra Astrid Arrieta Molinares, presidenta de la Asociación Colombiana de Psiquiatría (Redacción El Espectador, 2020) frente al problema de suicidio en Colombia, se anota que este permaneció durante muchos años con una tasa de de 4 por 100.000 habitantes, sin embargo, en los últimos años se incrementó a 5,9 por 100.000 habitantes. Además, explica que el país está mal adaptado, que tiene problemas de violencia, de pobreza y no existen oportunidades. En adolescentes de 15-19 años, el suicidio es la segunda causa de muerte entre niñas, y la tercera entre niños según informe de la Organización Mundial de la Salud (Redacción El Espectador, 2020, pp. 20-21). Bajo tal perspectiva, durante el último año se evidencia un aumento de expresiones y manifestaciones sociales como una forma de hacer un llamado de atención a los gobiernos de turno para diseñar mejores estrategias para remediar los problemas que aqueja a la humanidad, lo cual está poniendo en evidencia la vulnerabilidad de paz en el mundo.

Aunque las protestas masivas que tuvieron lugar en muchas ciudades del mundo en 2019 se formaron espontáneamente, no surgieron de la nada. La confianza en que los gobiernos o los mercados pueden ofrecer a la gente una oportunidad justa en la vida se ha debilitado en muchos países" (Shanmugaratnam, 2020, p. 6).

Esta perspectiva poco alentadora, debe llamar la atención tanto a los individuos como a las instituciones del Estado a pensar de manera mancomunada en la construcción de una ética de paz que se cuestione cuál es el compromiso verdadero que se debe tener con la humanidad y el planeta en general, donde el concepto de paz mundo no se pierda por el egoísmo de algunos grupos que ignoran la relación directa que tienen sus actuaciones presentes, con la injusticia social por la que atraviesa el mundo. 
Esas tensiones se manifiestan de diversas formas, según donde uno mire, pero reflejan realidades subyacentes. La movilidad social es obstinadamente reducida en muchos países, el crecimiento económico se ha desacelerado, los jóvenes ven menos perspectivas de buenos empleos y posibilidades de comprar una vivienda, y las brechas en la riqueza se han ampliado (Shanmugaratnam, 2020, p. 6).

Es claro que dichos problemas globales retan de una manera particular a los estudios de la paz para generar nuevos conocimientos en pro de la sostenibilidad y plausibilidad de la paz. Sin lugar a dudas en este nuevo pensar de un mundo posible, la educación jugará un papel trascendental, por ello es importante focalizar todos los esfuerzos en empoderar más la disciplina de la pazología ${ }^{2}$ en todas las instituciones educativas para impulsar la confianza en el futuro de las naciones.

Tal panorama refleja la urgente necesidad de aunar esfuerzos entre investigadores, academia, institucionalidad y comunidad por hacer de la paz algo más que un simple momento silenciado, algo más que una mera aspiración personal. Y es que la preocupación por mover la paz hacía el paradigma científico es una intención latente y que debe ser estudiado a profundidad, sin embargo, es entendible que el cambio paradigmático sea lento y osado (Sánchez Cardona, Forero y Duque, 2019, p. 173).

Es desde esta perspectiva, donde el artículo desea realizar algunos aportes a partir la Escuela Sociocultural de la Paz Pedagógica, en el cual se visualice al ser humano como creador de nuevos caminos hacia la solidaridad y la confianza en las innumerables posibilidades que posee para hacer las paces. Esta forma de aproximarse a la paz desde las potencialidades del

\footnotetext{
2 La pazología se refiere a los estudios de la evolución de la paz en todas sus dimensiones y diferentes contextos de vida: personal, familiar y social. Al respecto se recomienda las investigaciones de Sánchez Cardona (2017a; 2018b) quien ha analizado como la educación en varias facultades de derecho se ha centrado más en la violencia que la paz. De igual forma se recomienda revisar las investigaciones de Jiménez Bautista (2011; 2018), Marquardt (2017), Muñoz y Jiménez (2012), Martínez (2011), Adams (2014), entre otros.
} 
individuo que propone dicha escuela del conocimiento, ya se ha venido examinado en la historia de algunas comunidades y grupos sociales en Colombia, específicamente en los departamentos de Cundinamarca, Boyacá Antioquía (Castro, Duque, Sánchez Cardona, 2018) y Meta (Sánchez Cardona 2018d; Sánchez Cardona, Forero y Duque, 2019). Es por ello que, desde este enfoque para investigar la paz en diferentes escenarios sociales, se anhela seguir profundizando como una forma de aportar a la reconstrucción del tejido social a través de la firmeza en las bondades que posee la humanidad, para crear más ventanas y puentes de comunicación con los problemas que aqueja al mundo.

\section{Visiones de la Escuela Sociocultural de la Paz Pedagógica}

Pareciera que estamos tan acostumbrados a recibir todos los tipos de violencia, que cuando se presentan otros escenarios de convivencia donde la cultura de paz está más desarrollada creemos no merecer el valor propio y nos sentimos incomodos hasta en el ejercicio de expresar el afecto con un solo abrazo. Un colega expresaba:

-[...] profe me sentí tan raro e incómodo en escenarios donde me recalcaron que mi papel como docente era importante y donde propiciaron espacio para comunicar mi valía como persona, esto ya lo había olvidado ${ }^{3}$.

Necesitamos advertir que la cultura del maltrato y el no reconocimiento no debe legitimarse bajo ninguna circunstancia. Siguiendo esta perspectiva, la paz pedagógica tiene como misión utilizar estrategias pedagógicas basadas en el amor, la compasión, la esperanza y la escucha activa sobre sí mismos y hacia los otros para generar espacios que logren sensibilizar la capacidad de sentir de nuevo nuestras emociones que han sido anestesiadas por largos periodos de tiempo. Este fenómeno de adormecimiento en la capacidad de sentir y expresar

3 Conversación sostenida durante un encuentro de investigación entre colegas. 
la emoción, se ha observado tanto en las personas y comunidades al ser ignorados, excluidos(as), maltratados y vulnerado su derecho humano a la paz. Es así como la Escuela Sociocultural Paz Pedagógica, aspira a despertar e iluminar las capacidades humanas para enaltecer de nuevo la fe y el descubrimiento de aquella fuerza interior que poseen las personas, como una forma de propiciar la expresión de sus emociones que han sido silenciadas a través del tiempo.

Estos sentimientos de tantos rostros humillados deben tener un canal humano de expresión en la realidad actual, para ser escuchados y dignificados en torno al arte de hacer la paz. Es aquí donde la paz pedagógica se nutre con las vivencias, sueños, frustraciones y esperanzas de aquellos grupos, personas, minorías y comunidades que parecen ser insignificantes, pero que finalmente serán los importantes para el aprendizaje de la paz. La autora cree que una manifestación de toda esta emocionalidad escondida frente a la injusticia, ha acontecido en los fenómenos sociales ocurridos en el 2019 en varios países del mundo:

El contexto de las protestas que han ocurrido recientemente en Francia, España, Irak, Hong Kong, Libia, Bolivia y Chile, las diferencias abundan. Pero lo que parecen tener en común son las emociones que los motivan: rabia, inconformidad resentimiento, malestar, desesperación e indignación... Sobre todo, indignación, un rechazo casi instintivo a un estado de cosas injusto o inequitativo. La indignación puede estar movida por el resentimiento y el deseo de reconocimiento, algo que Francis Fukuyama ha interpretado como la causa del malestar de muchos ciudadanos con las democracias contemporáneas. (Garzón, 2020, p. 10)

La paz pedagógica no percibe la paz como una meta, sino como un proceso que se teje todos los días y se deshace con cada problemática de violencia que encontramos en las realidades cotidianas. Podremos expresar que, al contemplarse este constructo nuevo de paz, este actuaría como una llama ardiente 
que está siempre impulsando formas armónicas para coexistir con el otro y con todos los seres vivos. Adicionalmente, se alimenta del contacto directo con las personas y comunidades que han convivido con las expresiones de paz y de violencia, al igual hace reflexión en espacios de soledad donde se plantea nuevos paradigmas de aprendizaje, investigación y enseñanza de la paz en los escenarios permeados por la violencia. Por ello, la paz pedagógica quiere entender cómo desaprende el ser humano la violencia y cómo aprende nuevas formas pacificas de actuar en el mundo con una responsabilidad tangible frente a la paz mundo. Entre algunos de los lineamientos de la Escuela Sociocultural de la Paz Pedagógica se destacan:

1. Paz pedagógica- aprende con el otro y también en la soledad: con esta perspectiva se quiere denotar que el aprendizaje de los nuevos paradigmas de la paz se hace desde y con el otro. Se da un valor sobresaliente al reconocimiento de las verdades, subjetividades e imaginarios que tiene cada sujeto frente a los contextos de paz y/o violencia que se ha vivido. En este actuar desde y con el otro se construye un principio de verdad que aporta en el aprendizaje y pedagogía de la paz. Estas experiencias con las comunidades y grupos obligan a reflexionar en espacios de soledad a entender, construir y deconstruir nuevos paradigmas y teorías de paz centrado en los problemas reales de las personas y colectividades. En este enfoque el papel de expertos académicos en paz que no han tenido contacto con realidades sociales, posee poca cabida en esta construcción de saberes, pues definitivamente son las personas y grupos que sufren quienes juegan el rol de expertos, no los académicos mismos. En suma, el paradigma de conocimiento de paz gira del centro (académico) a la periferia.

2. Cuestiona nuevas formas de actuar hacia el concepto de paz mundo, la Escuela Sociocultural de la Paz Pedagógica desea investigar los desafíos que tienen los individuos para trascender los saberes y sentimientos pacíficos, hacia la búsqueda de soluciones de los problemas que agobian al mundo. Ente proceso 
reta a generar nuevos conocimientos hacia la viabilidad y sostenibilidad de la paz en del mundo, desde la perspectiva de compromiso que tiene cada uno con el cuidado de las relaciones armónicas en el planeta. Se actúa con reflexiones en torno a que lo se hace o se deje de hacer en cada escenario de vida afecta directa o indirectamente a todos por la interconectividad que se tiene con la vida misma. De este modo, se intenta aportar a la paz mundo de forma tangible, no solo desde la palabra sino también desde el actuar. Autores como Jiménez Bautista y Rueda Penagos plantean como símbolo del pacifismo "paz mundo" " , "[...] parte de la premisa y defensa del ser humano. De nuevo, el reto consiste en enfrentarse a nuestros miedos personales y gritar fuerte: debemos trabajar por un mundo de paz" (Jiménez Bautista y Rueda Penagos, 2012: 229).

Podría expresarse de una manera más clara que la paz pedagógica, al interior de la escuela sociocultural aspira a estar siempre sintonizada con el sufrimiento humano en los diferentes contextos psicosociales y desde estos escenarios pedagógicos se quiere reflexionar acerca de los obstáculos que impiden la realización del derecho humano a la paz. En este actuar con las pedagogías de la paz, se sensibiliza a cada individuo en un pensamiento holístico donde prima el pensar, sentir y actuar hacia una paz mundo en el que la indiferencia, el individualismo y la insensibilidad por el problema del otro no tienen cabida. Es en este proceso de consciencia individual y colectiva es donde se legitima a la ciudadanía y diferentes grupos activistas a confrontar constantemente a los dirigentes políticos en su rol que desempeña en pro de la paz social. En el contexto colombiano se ha criticado constantemente, el papel que ha tenido el Estado para cumplir los objetivos hacia una verdadera transformación pacífica.

\footnotetext{
4 Jiménez Bautista en investigaciones recientes de 2018, combina los términos de paz mundo y paz neutral como un aporte al nuevo paradigma pacifico. Ver cartografía de paces y cultura de paz en Temas actuales para la promoción de la cultura de paz, el estudio de conflictos y desarrollo (2018).
} 
La inercia de un estado paquidérmico y disfuncional aconseja también la reformulación. Más aun cuando la dirigencia del país no calculó los efectos de problemas sistémicos como la corrupción, la renuencia de altos funcionarios del Estado a autorreformarse, la baja calidad de la educación pública, los desmedidos privilegios de quienes viven del erario, la inequidad del sistema pensional, el impacto social de la inestabilidad, la informalidad laboral, la migración venezolana, un sistema tributario permanente, remendado e inequitativo (Garzón, 2020, p. 10).

La autora considera que esta inadecuada realización de las funciones de quienes representan al Estado ha ocasionado en la población sentimientos de desconfianza y falta de credibilidad por la paz, la cual es percibida como inalcanzable, débil o vulnerable, ya que ellos han impartido políticas que no han tenido grandes impactos en la calidad de vida de la ciudadanía y del planeta en general. Es por ello que la Escuela Sociocultural de la Paz Pedagógica tiene el deber de cuestionar, si el tema de la realización de la paz mundo, se debe dejar solo en manos de dirigentes políticos o es competencia de todos y cada uno de los individuos y grupos sociales. Sin embargo, también se tiene el conocimiento que estos bloqueadores sociopolíticos que hacen parte de la violencia estructural ${ }^{5}$ genera grandes miedos y visión de no futuro en los individuos y grupos que han sido excluidos de la sociedad, al respeto valdría la pena mencionar de nuevo a Jiménez Bautista y Rueda Penagos cuando precisan que:

Es el miedo que se ha convertido en nuestra diaria compañía, el pánico a asumir la responsabilidad individual, el abandono colectivo, todo ello nos obliga a reflexionar sobre esta nueva realidad y a buscar alternativas que compensen la pérdida de antiguas referencias en muchas personas; la ausencia de referencias éticas, tradicionales y valores, y el derrumbamiento de pautas culturales capaces

5 Concepto introducido por Johan Galtung en 1960, 1969 hace alusión a las injusticias e insatisfacción de las necesidades básicas humanas que se deben atender en una nación para vivir en paz. Retomado posteriormente por Fisas (2006) y Jiménez (2011). 
de frenar los actos violentos destructivos, etc., son los que se hacen presentes en la actualidad (Jiménez Bautista y Rueda Penagos, 2012: 230).

Es en estos contextos de angustia y poca claridad frente al futuro en que viven muchas personas en Colombia y el mundo entero, la Escuela Sociocultural de la Paz Pedagógica quiere hacer su aporte a la humanidad haciendo presencia $\mathrm{y}$ acompañamiento emocional a las colectividades, para desarrollar al interior de la cultura procesos de escucha reflexiva que ayuden a entender los sentimientos negativos que se generan en estas condiciones de vida. Este aprendizaje puede propiciar una red de apoyo y fortalecimiento de la capacidad de resiliencia en la sociedad, para impulsar nuevas formas de actuar hacia un mundo más justo y equitativo.

3. Comparte saberes con los otros a un mismo nivel de conocimiento (educando, educador, comunidad): Se desea apuntar que este aprendizaje de la paz pedagógica dentro de la Escuela Sociocultural de la Paz Pedagógica actúe desde un nivel horizontal donde el educando, educador y/o comunidad interactúen a un mismo nivel de jerarquía porque el conocimiento de la paz no está centralizado en un solo saber o grupos de personas. Este se encuentra disperso en todos los actores interesados en construir, debatir, discernir, interpretar y compartir las experiencias de paz, que terminarán irradiando estos saberes a los lugares donde las personas y grupos sociales han sido excluidos sistemáticamente. De esta manera, las redes de conocimiento de la paz quedan descentralizadas e impactan a las regiones y espacios de formación a las diferentes colectividades, que construyen y trabajan a diario para deconstruir la cultura de violencia y empoderar la paz en cada realidad.

4. Construye investigación desde el actuar cotidiano. El aprendizaje de la paz pedagógica surge desde las investigaciones cotidianas y desde allí se quiere desarrollar una masa crítica que cuestiona las formas de violencia emitidas desde las estructuras sociopolíticas de un contexto específico. Así pues, la 
paz pedagógica focaliza sus investigaciones en los individuos o grupos que están tejiendo nuevas formas de actuar en el mundo desde sus propios miedos, angustias y realidades. Ello permite enaltecer y visibilizar los saberes de aquellos que han sido ignorados por décadas en el país, fenómeno que ha obstaculizado su inclusión en pro del futuro de la paz.

En suma, centrando la atención en la investigación para la paz desde su cotidianidad, pero con una visión crítica ante el futuro de las realidades de violencia, se apunta a deslegitimar en las mentes de los individuos el continuismo del círculo de la pobreza y se reta a pensar y crear nuevos imaginarios y paradigmas de paz donde se conciba una mejorar calidad de vida que esté al alcance de todos y no solo se privilegie a una minoría de personas.

5. Fortalece y empodera a las juventudes hacia la construcción de un el tejido social con justicia e igualdad: Su lente de acción está dirigido a privilegiar las investigaciones que conduzcan a la búsqueda de estrategias que dirijan la edificación de formas de pensar y escenarios sociales donde se legitime la justicia social e igualdad de derechos para que todos puedan disfrutar de una paz duradera y sostenible. La paz pedagógica en este pensar, le hace una apuesta a las juventudes para que sean protagonistas de este cambio social (Sánchez cardona, 2015) y tenga un liderazgo mejor que los mismos políticos de turno.

En un mundo sin ideología que nos expliquen el pasado y nos proyecten hacia el futuro, muchos ciudadanos sienten miedo, desesperación y se identifican de manera muy frágil con el proyecto político nacional. No es casual que los jóvenes sean protagonistas de unos reclamos que se formulan precisamente en términos de deseos de un mejor mañana y que pongan sobre la mesa temas soslayados hasta ahora (Garzón, 2020, p. 10).

Se propone hacer un acompañamiento activo a esta población para motivarlo a seguir desempeñando su rol que ha cumplido para las grandes transformaciones de la sociedad 
(Sánchez Cardona, 2017b) e invitar desde sus potencialidades a no desfallecer en la construcción de un futuro, donde todos y cada uno tengan un papel activo en el arte de mejorar la convivencia con el otro. En este contexto se podría mencionar entre muchos ejemplos, el liderazgo de la joven Belga Amaya Coppens que, con solo de 25 años, afronta al presidente Ortega en Nicaragua quien, por defender la justicia en su país, ha caído dos veces presa y su lucha se ha convertido en el nuevo símbolo del movimiento social que pide un cambio político en el país centroamericano (Redacción Internacional, 2020, p. 5). Otro de los tantos ejemplos de jóvenes que han liderado cambios sociales más que los mismos gobiernos, es el caso de la activista sueca Greta Thunberng quien lucha por generar conciencia frente a las prácticas y políticas en torno al cambio climático.

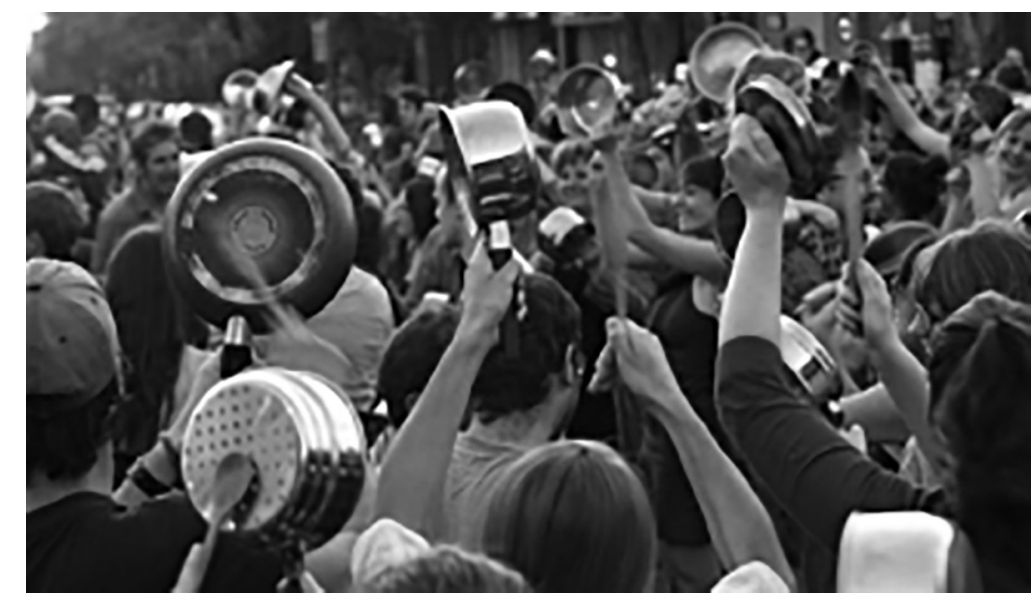

Con el cacerolazo se inaugura una nueva forma de protesta social en Colombia (Redacción La Guía Cundinamarca, 2019)

En este mismo sentido, puede evidenciarse en las expresiones simbólicas-políticas que se expanden a lo largo y ancho de los territorios como una forma de exteriorizar inconformidades, necesidades y urgencia de cambios, como el caso de las manifestaciones ocurridas entre el 21 de noviembre y el 15 de diciembre del 2019, en gran parte del territorio Colombia, 
muchas de ellas abanderaron el término cacerolazo como una opción de acción no violenta que pronuncia el cansancio, la desidia y el desinterés por continuar bajo un sistema político que profundiza la desigualdad, materializa la inequidad e incrementa -por acción y omisión- las horrores de una violencia que no abandona al país.

La autora advierte que la propuesta de la Escuela Sociocultural de la Paz Pedagógica debe incluir también en gran medida al profesorado, quien deberá ser formado tanto en el tema de la paz como en procesos humanos que faciliten la pedagogía de la paz en los educandos. Sin embargo, da un protagonismo central a la población joven en Colombia:

[...] quienes juegan un papel transcendental en la innovación de metodologías de paz en el manejo de los conflictos sociales. Son ello(as) indudablemente los que serán agentes de cambio y podrán impulsar los nuevos paradigmas de paz y esperanzas de vida para las futuras generaciones en pro de la construcción social de justicia y las relaciones pacíficas como el centro de atención de la humanidad (Sánchez Cardona, 2018c).

6. Teje la ética de la paz y no la mercantilización de la paz: uno de los grandes retos que aspira investigar la Escuela Sociocultural de la Paz Pedagógica, es rescatar la ética de la paz en las mentes de todos los ciudadanos para que se convierta en un gran vector que oriente el cambio de la cultura de la violencia por la cultura de paz. En este sentido, se quiere señalar que el tema de la paz en manos de los políticos de turno ha deteriorado su verdadero sentido, al hacer de ella un marketing de la paz, no una virtud fundamental para la construcción de una ética ciudadana tendiente a la práctica de virtudes, algunos autores han mencionado que:

Las virtudes dotan a los seres humanos de un sistema completo y plural de buenas formas de reconocimiento moral que facultan para la promoción de valores, de unión y accesorios que van desde el amor propio hasta 
el amor a los demás y el amor universal. (Muñoz y\& Molina Rueda, 2014: 59).

En esta misma dirección Linda Kavelin Popov en su texto $E l$ proyecto de virtudes (2000) resalta la virtud como una acción de fuerza, poder, capacidad y energía que permite:

Identificar y hacer conscientes a las personas de sus virtudes desde temprana edad, es una de las mejores formas para estimular voluntariamente sus potencialidades e incentivar su utilización cada vez que sea necesario (Kavelin Popov, 2000, p. 5).

La investigadora Popov (2000), ha aplicado en varios contextos sociales su teoría de las virtudes en pro de la cultura de la paz. Por ello se quiere retomar este concepto para impulsar la visión de una ética de paz que ilumine todas acciones en la tierra. En este misma perspectiva la autora, está de acuerdo con el pensamiento del investigador Alemán Marquardt (2019) quien afirma que ninguna paz en el contexto colombiano es imposible y que para ello:

[...] es trascendental trabajar en el camino de las mentalidades entrenadas durante la Guerra de los 67 años que nadie puede malentender eufemísticamente como supuestas tradiciones culturales valiosas. El método clave es la educación para la paz, transmitiendo valores de la convivencia autocontrolada, cooperativa, constructiva y respetuosa. (p. 45)

De esta manera, la paz debe empoderarse como virtud en las instituciones educativas para que se desarrolle una masa crítica de abajo hacia arriba que logre permear de forma lenta y segura las estructuras de violencia que han sido construidas culturalmente a lo largo de la historia. En esta dirección, es posible señalar el caso del profesor Londinense Alex Beard, quien visitó cerca 20 países buscando prácticas pedagógicas exitosas. Afirma que no que no existe un método perfecto de educación, no obstante, reconoce la importancia de trabajar en la sociedad en temas de responsabilidad compartida 
No creo que haya una educación perfecta. Una de las causas de que a Finlandia y Singapur les vaya tan bien en las Pruebas Pisa no son los colegios, son los valores y las actitudes en la sociedad. En Finlandia todos aman la educación, hay una responsabilidad compartida de todos. Ese es uno de los puntos en común entre países con prácticas pedagógicas exitosas (Taborda, 2020, p. 7).

Desde este horizonte, la paz pedagógica cree de nuevo que las generaciones jóvenes con el aprendizaje de una ética discusiva y argumentativa puede orientarse en la defensa del derecho a la educación para la paz y con este horizonte, se puede lograr avivar la compresión y la importancia de generar una exigencia a la sociedad hacia una ética de la paz, para sacar de los imaginaros aquella idea de paz que se ha promulgado como un comodín y bandera en los últimos años por las estructuras políticas de turno, con el ánimo solo y exclusivamente de ganar seguidores en sus campañas políticas. Uno de los tantos casos que evidencia este pensamiento, se observa en municipio del Tambo, departamento del Cauca- Colombia, donde existen 12.000 víctimas del conflicto armado y sus pobladores aún no reciben ninguna reparación para dar cumplimiento al acuerdo de Paz alcanzado entre el Gobierno colombiano y el grupo guerrillero Fuerzas Armadas Revolucionarias de Colombia- Ejercito Popular (FARC-EP):

El profesor José Antonio Ante dice que la Ley de Víctimas le prometió al Colegio Agroindustrial, hace cuatro años, un sistema de producción pecuaria que consistía en 500 gallinas, 200 pollos de engorde, cinco novillas preñadas, 20 colmenas de abejas, 10 conejos y apoyo para cultivos de plantas medicinales. Todavía están esperando. En pocas palabras, las víctimas son el propósito de todos los políticos, pero cuando llegan a los cargos las promesas de campaña se olvidan (Umaña, 2020, p. 7).

Tales ejemplos exponen el peso mediático que ha tenido el tema de la paz en los últimos gobiernos, convirtiéndola en una bandera o propuesta de campaña y no en una política o 
meta-finalidad (Marquardt, 2017) del Estado, ello deriva en convertir la paz en un producto volátil y mercantil que es prioritario para algunos e insignificante para otros. (Rivas, 2016).

7. Se sitúa desde el interior como fuente de pedagogía-aprendizaje: La fuente principal del aprendizaje de la paz pedagógica es la vivencia interior que cada individuo y grupo ha tenido de las paces en sus diferentes escenarios de vida, donde se desea remover todas las capacidades de las comunidades, educandos y educadores que están invisibilizando la esencia misma de la paz. Esta paz pedagógica se instala al interior de cada uno, para hacer resonar las experiencias de vida de la paz que se ha generado en diferentes momentos y desde ahí se pretende posibilitar espacios de expresión social que ayude a otros en la búsqueda de la paz, para que sea el motor que posibilita los cambios hacia un futuro mejor de la sociedad. Por ello propone la autora la pertinencia de "desarrollar contenidos de una formación integral, donde la educación sea vista como un proceso humanizador y democrático "como crecimiento interior del individuo", fundado en la constitución de ciudadanos para la paz, la convivencia y los derechos humanos." (Sánchez Cardona, 2017b)

En esta dirección, en cada encuentro con las personas o comunidades se trata de desplegar procesos humanos que posibiliten la búsqueda de tesoros escondidos para explorar cuidadosamente aquellos indicios donde puede existir, individual o socialmente, la perla preciosa de la paz. Para ello la Escuela Sociocultural de la Paz Pedagógica explora procesos humanos de concordia en los grupos tanto a nivel del pensar, sentir y hacer en la historia de cada uno. En este camino se ha encontrado un común denominador y es que estos destellos de luz de paz han sido frecuentes en la vida de las personas, sin embargo, se ha opacado su exteriorización debido a la multidimensionalidad de la violencia que impide que emerjan dichas experiencias de vida.

La Escuela Sociocultural de la Paz Pedagógica ha ratificado que el ser humano tiene el potencial de la paz que al hacerla consciente y socializarse nos identificamos como iguales y 
podemos luchar por el objetivo de la realización de la misma. De nuevo queremos subrayar que esta pedagogía de la paz se construye desde las vivencias de cada una y este aprendizaje es único. Con esta visión, la fuente de enseñanza no es la teoría misma de la paz ni las fórmulas prediseñadas, sino la experiencia de vida de cada persona como actor principal del proceso de paz que nace de su interior, desde allí se teje la pedagogía propia de la paz y sus conceptos. Se hará más visible a nivel social y se multiplicara si las instituciones sociopolíticas posibilitan escenarios para su desarrollo (Nussbaum, 2016).

8. Esta en continua construcción y se reinventa todos los días: la paz pedagógica está en continua evolución, no es lineal ni pretende aplicar igual metodología ni construcciones de paz en todos los contextos de la misma manera, ya que tiene claro que cada problema se desarrolla de forma diferente en cada realidad. Se nutre de la creatividad y aprendizajes nuevos que cada grupo ha tenido en ambientes de violencia y/o de paz. Se reinventa constantemente para lograr entender las dificultades que tienen las comunidades en el día a día del quehacer de la paz, donde cada experiencia entorno a la violencia es única como cada aprendizaje de armonía lo es en su momento. Los contenidos de la paz no son el foco de accionar de la pazpedagógica, sino los procesos y vivencias que ha tenido cada persona y/o comunidad de la cual siempre habrá una construcción nueva de cultura y ella será única e irrepetible. Partiendo de estos aprendizajes la Escuela Sociocultural de la Paz Pedagógica quiere hacer una apuesta continua a dinamizar nuevas formas de actuar en el concepto de paz mundo. Por tanto, ver la paz bajo la visión de mundo, expone que no seremos ajenos a las problemáticas presentes y futuras de la paz, tanto en las interacciones con nosotros mismos, los otros y el medio ambiente.

9. Focaliza procesos de escucha reflexiva y de compasión en las interacciones. Una de las estrategias para potencializar los procesos personales de paz de cada individuo y colectividad, es la escucha reflexiva de todos y cada uno de los participantes 
durante los procesos de socialización de problemáticas en la realización del derecho humano a la paz en sus escenarios de vida. Con esta estrategia la paz pedagógica desea concienciar en la compresión de la historia de vida del otro por medio de la compasión ${ }^{6}$ la cual no pretende cambiar a nadie ni entrar en juicios de valor de las realidades del otro. El psicólogo Marshall Rosenberg $(2011 ; 2018)$, en sus investigaciones de manejo de conflicto, ha desarrollado el proceso de escuchar para facilitar metodologías no violentas en los conflictos.

La Comunicación No Violenta (CNV) nos enseña a observar cuidadosamente y detectar conductas y situaciones que nos afectan [...] al centrarnos con clarificar en lo que observamos, sentimos y necesitamos, en lugar de dedicarnos a diagnosticar y juzgar, se descubre cuán profunda es nuestra compasión. Dado que la CNV se centra principalmente en escuchar con atención no sólo a los demás, sino también a nosotros mismos, propicia el respeto y la empatía y engendra un deseo mutuo de darnos de todo corazón (Rosenberg, 2000: p. 16).

Es importante clarificar que, en la comunicación no violenta, el uso de la escucha reflexiva, es fundamental para lograr percibir los puntos de vista de los otros sin desconectarnos de nuestros sentimientos, pero tratando en la medida de lo posible alejar los juicios de valor e interpretaciones de las actuaciones de las personas (Sánchez Cardona, 2018c, p. 156). Este proceso se puede equiparar con la idea de observar sin juzgar y ello facilita el silencio de la mente (Fernández, 2005, pp. 391-400).

En este sentido se hace un esfuerzo en despertar los sentimientos de solidaridad que se percibe al escuchar y entender el dolor humano sufrido sistemáticamente a causa de la injusticia social. El objetivo que persigue esta estrategia de escucha reflexiva, es meditar sobre los procesos interiores que se generan en cada

\footnotetext{
6 Al respecto se puede consultar el texto de la ciencia de la compasión de García Campayo, Cebolla y Dermazo (2016), donde desarrollan claramente el concepto y proponen una terapia útil para desarrollar la virtud en el ser humano.
} 
uno, como producto del contacto con los excluidos socialmente y desde este sentir promover conductas en el compromiso por cambiar el rumbo de la inequidad social en el mundo.

Como se manifestó en el apartado inicial, en países como Colombia pareciera que la interacción con los escenarios de violencia por largos periodos de tiempo nos ha anestesiado a las manifestaciones de afecto para con nosotros mismos y los demás. Hemos interiorizado la cultura de la exclusión y cuando se nos abren las puertas para la participación en el futuro de la humanidad, pareciera que se va perdiendo lentamente en algunos grupos esta capacidad de asumir el rol activo en la sociedad.

La autora ha encontrado en sus investigaciones (2018a), que las manifestaciones de violencia en la vida de las personas han dejado tantas secuelas, que se percibe con frecuencia sentimientos de incomodidad y molestia en los espacios donde nos brindan la posibilidad de tener una igualdad de derechos. Pareciera que la auto- estima y la auto- valía al estar maltratada en la historia de vida de cada uno, ha dejado cicatrices profundas en las relaciones socioafectivas. Por consiguiente, se puede deducir que ahora más que nunca se necesita nuevas y mejores enseñanzas cada vez más poderosas para movilizar el afecto y sinergia de los individuos en pro de la paz.

En recientes trabajos de campo con algunas comunidades de mujeres -en la ciudad de Pasto, departamento de Nariño, Colombia-, que han sufrido durante años maltrato y exclusión en diversos escenarios, se ha evidenciado la gran dificultad en este tipo de poblaciones para encontrar y reconocer sus valías como mujer, madre y lideresa social. Ellas han expresado -durante los encuentros de paz realizados con la Corporación Sentire ${ }^{7}$ en

\footnotetext{
7 La autora es una de las cofundadoras de la corporación Sentiré creada en el 2018, una organización sin ánimo de lucro que propicia escenarios en el área socio-educativa y de investigación tanto para personas y grupos que han sido excluidos sistemáticamente. Así mismo la corporación, vincula a los empresarios y sociedad en general, a través de procesos humanos donde se active la virtud de la compasión y el sentir, desde lo afectivo, cognitivo y motivacional como una forma de contribuir a la cultura de la paz.
} 
diciembre del 2019 y enero de 2020- enormes dificultades para examinar sus virtudes, debido a que gran parte de sus vidas se ha enaltecido culturalmente la sumisión frente al reconocimiento de sus potencialidades. Pareciera que han interiorizado tanto su papel y rol de sumisión social que han olvidado pensarse como sujetas activas para las transformaciones de sus regiones. Podría pensarse que este fenómeno de falta de reconocimiento personal de sus propias valías, se ha aprendido culturalmente pues no han sido educadas para mirarse y valorarse ellas mismas debido a la continua supresión de sus derechos impartida por las relaciones de poder patriarcales y opresoras. En estos contextos la Escuela Sociocultural de Paz Pedagógica, le compete avivar la llama de las virtudes de las poblaciones que han sido suprimidas a causa de la violencia utilizando estrategias que propicien procesos de auto-reconocimiento de las virtudes de la paz, las cuales deberán fluir desde el interior de las personas y expandirse hacia el afuera como un medio para activar su rol en los verdaderos desarrollos personales que impactaran indudablemente en los progresos de sus regiones.

En esta misma dirección la Escuela Sociocultural de la Paz Pedagógica, propicia conocimientos que ayude a la sensibilización a la capacidad de amar y la valía propia, para restablecer la auto-confianza (autoeficacia) en ellos mismos y de esta manera, romper con el círculo vulnerable de admisión de los maltratos que han sido legitimados por largos periodos de tiempo en sus vidas. De esta manera se puede despertar la capacidad de indignación ante la violencia creando una masa crítica ante la violación de sus derechos y se crear ambientes de bondades y virtudes de auto-reconocimiento donde se focalice los esfuerzos en reconocer sin temores, ni humildad sus fortaleces de paz que poseen tanto a nivel individual como social. Este trabajo desde su interior contribuye a empoderarlos en su nuevo papel en pro de la cultura de la paz en sus entornos y disminuye la perspectiva de ser vistas como disminuidas, suprimidas y opacadas socialmente. 
En suma, los apartados desarrollados en este articulo desea acercarse desde otras formas de saber a las problemáticas sociopolíticas que están impidiendo la realización del derecho humano a la paz en la sociedad, con el animo de aportar de forma proactiva en el desarrollo de la sostenibilidad de paz en la nación.

\section{Conclusiones}

La necesidad de ejecutar propuestas como la Escuela Sociocultural de la Paz Pedagógica permitirá arrebatar la paz de las manos de los actores políticos, violentos o los grandes centros de poder, lo que resultará en una construcción pacífica mancomunada donde el foco de atención sea la periferia.

En las nuevas aproximaciones de investigación para la paz, se precisa construir pedagogías del saber desde el conocimiento de los grupos y personas que han sido sistemáticamente excluidas, son desde estos saberes donde se nutre y se debe enaltece la verdadera edificación de paz en la sociedad.

La aspiración de la paz pedagógica, es sensibilizar a la sociedad en general en pro de una consciencia individual y colectiva donde el pensamiento, sentimiento y actuación de cada uno, contribuya a restructurar el tejido social hacia la concepción de paz mundo.

En la construcción de la cultura de la paz, es fundamental acompañar a las juventudes en la construcción de nuevos paradigmas de convivencia que legitime y exija a la sociedad una ética de la paz, basada en la justicia social e igualdad de derechos para que todos puedan disfrutar de una paz duradera y sostenible en el planeta.

\section{Bibliografía}

Adams David (2014): Cultura de paz: una utopía posible. México D.F, Editorial Herder.

Ansembourg, D. y Van Reybrouck, D. (2017). La paz se aprende. Madrid: Arpa. 
Castro, S., Sánchez Cardona, M. y Duque, C. (2018). Narrando paz ando. Iniciativas exitosas de paz en Cundinamarca, Boyacá y Antioquia, 2005-2013, Bogotá: Universidad Nacional

Fernández Herrería, A. (2005): Mundo emocional y silencio a través de la observación lúcida. En Actas V Congreso Internacional de Filosofía de la Educación (pp. 391-400). Madrid: Dykinson. Fisas Vicenc (2006): Cultura de paz y gestión de conflictos. Barcelona: Editorial Icaria.

Garcia Campayo, J., Cebolla, A. y Dermazo, M. (coords.). (2016). La ciencia de la compasión más allá del mindfulness. Madrid: Alianza.

Garzón, I. (2020). Marchas, cacerolas y mayorías silenciosas, en Periódico El Espectador, 17 de enero.

Galtung, J. (1960). The Strategic of conflict, Cambridge: Harvard University Press.

- (1969). Violence, Peace, and Peace Research, in Journal of Peace Research, 6(3). 167-19.

Instituto Nacional de Medicina Legal.(2019). Boletín Estadístico[2019]. https://www.medicinalegal.gov.co/documents/20143/349426/ diciembre-2019.pdf/320ad04c-7c85-287f-804a-a49b1031d9f3

Jiménez Arenas, Juan y Muñoz, Francisco. (2012). La Paz, partera de la historia, en Jiménez Arenas, Juan y muñoz, Francisco (eds). La Paz, partera de la historia. Universidad de Granada. Granada. (13-68)

Jiménez Bautista, F. (2018). Cartografía de paces y cultura de paz, en Gómez Collado, M (Coord.). Temas actuales para la promoción de la Cultura de paz, el estudio de Conflictos y el Desarrollo, Toluca, Universidad Autónoma del Estado de México.

- (2011). Racionalidad Pacífica. Una introducción a los Estudios para la paz. Madrid: Editorial Dykinson.

Jiménez Bautista, F. y Rueda Penagos, Z. (2012). Hacia un paradigma pacífico: la paz mundo, la paz compleja y la paz neutra, en Ra-Ximbai. 8(3). 223-243.

Marquardt, B. (2017). Ius Contra Bellum -la promoción del potencial humano a la paz-, Bogotá: Ibáñez.

- (2019). La paz territorial como paz judicial y justicia social reflexión sobre las oportunidades de dirigir el camino de 
Colombia al posconflicto material, en Marquardt, B., Martínez, J. y Sánchez Cardona, M. (eds.). Paz territorial y paz pedagógica. Revisión de tres años de posacuerdo en una sociedad dividida, Bogotá: Ed. Ibáñez.

Marquardt, B., Martínez, J. y Sánchez Cardona, M. (eds.). (2018). Paz territorial y tierras. Una mirada crítica frente a los acuerdos de la Habana. Bogotá: Ibáñez.

Mesa Jesús (2020): Ser niño en Honduras y no desaparecer en el intento, en Periódico El espectador, 2 febrero.

Muñoz, F y Molina Rueda, B. (2014). Virtudes clásicas para la paz. En F. Muñoz, y B. Molina Rueda (eds.), Virtudes clásicas para la paz. Granada: Universidad de Granada.

Nussbaum Martha (2016): Crear capacidades: propuesta para el desarrollo humano. Bogotá. Editorial planeta

Taborda, C. (2020). El hombre que recorrió el mundo para transformar la educación, en Periódico El Espectador 28 de enero.

Redacción El Espectador (2020). El suicidio no debe entenderse como falta de carácter, en Periódico el Espectador, 2 de febrero.

Redacción Internacional. (2020). La joven belga que se enfrenta a Ortega en Nicaragua, en Periódico El Espectador, 29 de enero.

Redacción La Guía Cundinamarca. (2019). "Con el cacerolazo se inaugura una nueva forma de protesta social en Colombia": sociólogo Fabián Sanabria, en La Guía Cundinamarca, 23 de noviembre. Recuperado de: https://laguiacundinamarca.com/ noticia/23618/con-el-cacerolazo-se-inaugura-una-nueva-formade-protesta-social-en-colombia-sociologo-fabian-sanabria

Rosenberg, M. (2000). Comunicación no violenta. Barcelona, Ediciones Urano.

- (2011): Resolver los conflictos con Comunicación No Violenta. Barcelona: Editorial Acanto S.A

- (2018): Serpaz en un mundo en Conflicto. Barcelona: Editorial Acanto S.A

Rivas, J. (2016). Los acuerdos de paz: ¿tema central de los partidos en la campaña presidencial en Colombia?, en Ciencia Politica, 11(21), 365-396.

Sánchez Cardona, M. (2015). Potencialidades de las generaciones jóvenes en el fortalecimiento del derecho humano a la paz, En Marquardt, B. (ed.). El estado constitucional de los valores $l$. 
Anuario $V$ del grupo de Investigación CC Constitucionalismo comparado. Editorial Ibáñez.

- (2016). Paradigmas de paz y potsconflicto en Colombia, en Marquardt, B. (Coord.). Paz a través del derecho y de la constitución, constitucionalismo científico, Bogotá: Ed. Ibáñez.

- (2017a). El aprendizaje de la paz en el derecho: perspectivas a partir de la labor del profesorado, en Debates Contemporáneos sobre Administración de Justicia Penal en los Albores del Posconflicto en Colombia Tomo I.

- (2017b). Hacia una propuesta de escuela sociocultural de la paz pedagógica en el postconflicto en Colombia, en Ballesteros M. (Comp.). Justicia Constitucional Tomo I. Bogotá: Ed. Ibáñez.

- (2018a). Educación para la cultura de paz: una aproximación psicopedagógica. Bogotá: Ed. Universidad Santo Tomas.

- (2018b). La universidad un escenario de paz y convivencia, en Muriel, J., Checa, M. y Krüggeler, T. (eds.). Paz, Reconciliación y Justicia Transicional. Bogotá: Editorial Pontificia Universidad Javeriana.

- (2018c). La escuela sociocultural de la paz pedagógica en Colombia, en Revista de Cultura de Paz, 2, pp. 109-124.

- (2018d). La paz territorial más allá del acuerdo de La Habana, en Marquardt, B., Martínez, J. y Sánchez Cardona, M. (eds.). (2018). Paz territorial y tierras. Una mirada crítica frente a los acuerdos de la Habana. Bogotá: Ibáñez.

- (2019). Cuatro mitos y una propuesta para construir la paz, en Revista CoPaLa, 4(8), pp. 95-108.

Sánchez Cardona, M., Forero, J. y Duque, M. (2019). De la Habana a la tierra: voces y pensamientos de las comunidades del departamento del Meta, en Marquardt, B., Martínez, J. y Sánchez Cardona, M. (eds.) Paz territorial y paz pedagógica. Revisión de tres años de posacuerdo en una sociedad dividida, Bogotá: Ed. Ibáñez.

Shanmugaratnam, T. (2020). Pensadores 2020: protestas sociales no cederán si no se gobierna desde el centro, en Periódico $E l$ Espectador, 22 de enero.

Umaña, O. (2020). Los muertos y las promesas incumplidas en el Tambo, Cauca, en Periódico El Espectador, 21 de enero. 